Acta Crystallographica Section A

Foundations of Crystallography

ISSN 0108-7673

Received 29 October 2002

Accepted 27 June 2003

(C) 2003 International Union of Crystallography Printed in Great Britain - all rights reserved

\section{The maximum-entropy method in superspace}

\author{
Sander van Smaalen, ${ }^{*}$ Lukáš Palatinus and Martin Schneider
}

Laboratory of Crystallography, University of Bayreuth, 95440 Bayreuth, Germany. Correspondence
e-mail: smash@uni-bayreuth.de

One of the applications of the maximum-entropy method (MEM) in crystallography is the reconstruction of the electron density from phased structure factors. Here the application of the MEM to incommensurately modulated crystals and incommensurate composite crystals is considered. The MEM is computed directly in superspace, where the electron density in the $(3+d)$ dimensional unit cell $(d>0)$ is determined from the scattering data of aperiodic crystals. Periodic crystals $(d=0)$ are treated as a special case of the general formalism. The use of symmetry in the MEM is discussed and an efficient algorithm is proposed for handling crystal symmetry. The method has been implemented into a computer program BayMEM and applications are presented to the electron density of the periodic crystal $\mathrm{NaV}_{2} \mathrm{O}_{5}$ and the electron density of the incommensurate composite crystal $(\mathrm{LaS})_{1.14} \mathrm{NbS}_{2}$. The MEM in superspace is shown to provide a model-independent estimate of the shapes of the modulation functions of incommensurate crystals. The discrete character of the electron density is found to be the major source of error, limiting the accuracy of the reconstructed modulation functions to approximately $10 \%$ of the sizes of the pixels. MaxEnt optimization using the Cambridge and Sakata-Sato algorithms are compared. The Cambridge algorithm is found to perform better than the Sakata-Sato algorithm, being faster, always reaching convergence, and leading to more reliable density maps. Nevertheless, the Sakata-Sato algorithm leads to similar density maps, even in cases where it does not reach complete convergence.

\section{Introduction}

The maximum-entropy method (MEM) is a powerful tool for model-free image reconstruction in many scientific applications (von der Linden et al., 1998). The MEM has been applied in crystallography in several ways, including the determination of the phases of the structure factors and the extraction of reflection intensities from powder diffraction data (Gilmore, 1996). Furthermore, the MEM has been used as an alternative method to multipole refinements, with the purpose to compute accurate electron densities that reveal the bonding electrons. After the first promising applications in this field (Collins, 1982; Sakata \& Sato, 1990), several warnings concerning the reliability and possible pathologies of the method appeared (Jauch, 1994; de Vries et al., 1996).

In a previous publication (Palatinus \& van Smaalen, 2002), we have investigated these problems and it was demonstrated that the MEM with a uniform prior leads to artifacts that are larger than the differences between the true electron density and the electron density of a procrystal that is based on spherical atoms. A new constraint was proposed that is based on the higher-order central moments of the distribution of
$\left[F^{\mathrm{MEM}}(\mathbf{H})-F^{\text {obs }}(\mathbf{H})\right] / \sigma(\mathbf{H})$. Although this constraint reduced the sizes of the artifacts, the resulting density still is not accurate enough to determine the effects of chemical bonding on the electron densities. The use of a non-uniform prior density appeared to be necessary (de Vries et al., 1994; Papoular et al., 2002).

A different goal of the MEM is to describe the effects of disorder or anharmonic temperature movements on the electron density. These effects are larger than the effects of the chemical bonding. It has been demonstrated in a series of publications that disorder (Dinnebier et al., 1999; Wang et al., 2001) and anharmonic displacements (Kumazawa et al., 1995; Bagautdinov et al., 1998) can be determined by the MEM.

A similarly large effect on the electron density is provided by the displacements of the atoms in aperiodic crystals out of their basic structure positions. The shifts are characterized by modulation functions that have arbitrary shapes. However, only one or a few parameters of the Fourier expansions of these functions can be obtained from structure refinements employing the superspace formalism (de Wolff et al., 1981; van Smaalen, 1995). An ab initio determination of the shapes of the modulation has turned out to be difficult. 
A model-independent reconstruction of the shapes of the modulation functions of aperiodic crystals is possible with the MEM in superspace. The application of the MEM to the generalized electron density in superspace was independently proposed by Papoular et al. (1991) and Steurer (1991). Weber \& Yamamoto (1997) have employed the MEM in a procedure to determine the shapes and positions of the occupation domains in five-dimensional (5D) superspace of the decagonal quasicrystal $\mathrm{Al}_{70} \mathrm{Mn}_{17} \mathrm{Pd}_{13}$. However, most publications report applications of the MEM to special two-dimensional (2D) and three-dimensional (3D) sections of the superspace structure of quasicrystals, thus circumventing the full $n \mathrm{D}$ problem $[e . g$. see Haibach et al. (2000) and Perez-Etxebarria et al. (2001)]. The application of the MEM to an incommensurately modulated structure has been considered only once, however without a quantitative analysis of the resulting electron density (Steurer, 1991).

In the present manuscript, a critical evaluation is made of the MEM as it can be applied to incommensurately modulated structures and incommensurate composite crystals. An efficient algorithm is used for the Fourier transform in $n \mathrm{D}$ superspace (Schneider \& van Smaalen, 2000), and a general algorithm is presented for handling symmetry as it applies to the reflection data as well as the electron density in arbitrary dimensions. These algorithms have been incorporated into a computer program, BayMEM, for MaxEnt calculation on periodic density functions in arbitrary dimensions. BayMEM can be used for full superspace MaxEnt calculations on problems of sufficient sizes, and thus it overcomes the previously noted problems of too large memory requirements for unrestricted MaxEnt calculations in superspace (de Boissieu et al., 1991; Yamamoto et al., 1996; Perez-Etxebarria et al., 2001)

BayMEM has been applied to the $(3+1) \mathrm{D}$ structure of the incommensurate composite crystal $(\mathrm{LaS})_{1.14} \mathrm{NbS}_{2}$. With calculated structure factors of the best structure model employed as 'experimental' data in the MaxEnt procedure, it is shown that the MEM can quantitatively reconstruct the modulation functions. The sources for the remaining discrepancies between model and reconstructed modulation functions are analysed, and it is shown that these discrepancies are mainly determined by the resolution of the discrete electron density (by the pixel size). These conclusions are confirmed by the application of BayMEM to the experimental data of $(\mathrm{LaS})_{1.14} \mathrm{NbS}_{2}$.

3D space is but one realization of arbitrary dimensions, and BayMEM allows for MaxEnt computations of periodic crystals too. Thus, we have employed BayMEM to study the electron densities of the periodic crystal structures of oxalic acid dihydrate (Palatinus \& van Smaalen, 2002), silicon and $\mathrm{NaV}_{2} \mathrm{O}_{5}$. Both the Sakata-Sato algorithm (Sakata \& Sato, 1990) and the Cambridge algorithm (Skilling \& Bryan, 1984) have been implemented in BayMEM. The results obtained by these two methods are compared. The performance of BayMEM is compared with the performance of the $M E E D$ program, the latter being restricted to three-dimensional space (Kumazawa et al., 1993).

\section{Theory}

\subsection{The entropy in superspace}

The crystal structures of aperiodic crystals are described by the superspace formalism (de Wolff et al., 1981; van Smaalen, 1995). Within this theory, $d$ additional dimensions are introduced that are orthogonal to the three dimensions of physical space. Together they define $(3+d) \mathrm{D}$ superspace. Bragg reflections of aperiodic crystals can be indexed with $(3+d)$ integers $\left(h_{1}, \ldots, h_{3+d}\right)$ with respect to a set of $(3+d)$ reciprocal vectors:

$$
\mathbf{H}=\sum_{k=1}^{3+d} h_{k} \mathbf{a}_{k}^{*} .
$$

They can be considered to be the projections of reciprocallattice points in superspace along the $d$ additional dimensions onto 3D physical space. In this way, a reciprocal lattice is defined in superspace.

The superspace theory shows that the electron density of the crystal can be obtained as a 3D section of a generalized electron density in superspace perpendicular to the $d$ additional dimensions. The generalized electron density $\rho_{s}\left(\mathbf{x}_{s}\right)$ is a periodic function of the superspace coordinates $\mathbf{x}_{s}$ according to the direct lattice corresponding to the reciprocal lattice defined by the reflections. This lattice is skew with respect to $3 \mathrm{D}$ space, resulting in a crystal structure that lacks 3D translational symmetry.

The entropy functional for an aperiodic crystal should be defined in direct analogy to the entropy for periodic crystals by employing $\rho_{s}\left(\mathbf{x}_{s}\right)$ instead of the electron density itself (Papoular et al., 1991; Steurer, 1991). The appropriateness of this definition is supported by the observation that properties that depend on the electron density itself can be computed in superspace (e.g. the structure factor and interatomic distances), while quantities that depend on derivatives of the density cannot be generalized towards superspace (e.g. lattice vibrations). The entropy is a functional of the density and it does not depend on derivatives of this quantity.

The generalized electron density in the $(3+d) \mathrm{D}$ unit cell is discretized on a grid of

$$
N_{\text {pix }}=N_{1} \times \ldots \times N_{3+d} \text { pixels }
$$

(Schneider \& van Smaalen, 2000). The discretized electrondensity function is then defined by the values of $\rho_{s}\left(\mathbf{x}_{s}\right)$ on this grid,

$$
\rho_{k}=\rho_{s}\left(\mathbf{x}_{s}(k)\right),
$$

where $k$ enumerates the $N_{\text {pix }}$ pixels according to the scheme defined in Schneider \& van Smaalen (2000). Then the entropy is defined as

$$
S=-\sum_{k=1}^{N_{\text {pix }}} \rho_{k} \log \left(\rho_{k} / \tau_{k}\right),
$$

where $\tau_{k}$ are the values of the reference density.

The MEM defines $\rho_{s}^{\mathrm{MEM}}\left(\mathbf{x}_{s}\right)$ as the generalized electron density that maximizes $S$, while it fulfils a series of constraints of the form $C_{j}=0\left(j=1, \ldots, N_{c}\right)$. The constraints include the 
fit to the experimental data. This can be the so-called $F$ constraint, defined by (Sakata \& Takata, 1996)

$$
C_{F}=-1+\frac{1}{N_{F}} \sum_{i=1}^{N_{F}}\left(\frac{\left|F_{\mathrm{obs}}^{i}(\mathbf{H})-F_{\mathrm{MEM}}^{i}(\mathbf{H})\right|}{\sigma^{i}(\mathbf{H})}\right)^{2},
$$

where the summation runs over all independent measured structure factors $N_{F} . F_{\text {obs }}(\mathbf{H})$ are the observed structure factors including phases and $\sigma(\mathbf{H})$ are their standard uncertainties. The structure factors $F_{\mathrm{MEM}}(\mathbf{H})$ are obtained by numerical Fourier transform from the trial electron density $\left\{\rho_{k}\right\}$. Other choices for the constraint on the data are possible too. In particular, the $F$ constraint can be replaced by a constraint on higher-order moments of the distribution of residuals of structure factors. This $F_{n}$ constraint is defined as (Palatinus \& van Smaalen, 2002)

$$
C_{F_{n}}=-1+\frac{1}{m_{n}} \frac{1}{N_{F}} \sum_{i=1}^{N_{F}}\left(\frac{\left|F_{\mathrm{obs}}^{i}(\mathbf{H})-F_{\mathrm{MEM}}^{i}(\mathbf{H})\right|}{\sigma^{i}(\mathbf{H})}\right)^{n},
$$

where $m_{n}$ is chosen so as to make the expectation value of $C_{F_{n}}$ equal to zero in the case of a Gaussian distribution. The original $F$ constraint is equal to the $F_{2}$ constraint in (6).

The second constraint is the normalization of the electron density

$$
\left(V / N_{\text {pix }}\right) \sum_{k=1}^{N_{\text {pix }}} \rho_{k}-N_{\text {el }}=0,
$$

where $N_{\mathrm{el}}$ is the number of electrons and $V$ is the volume of the unit cell. The actual value of $N_{\mathrm{el}}$ is determined by the scale of the experimental data, which can be easily derived from the superspace refinement.

\subsection{Symmetry of the electron density}

The electron density is required to obey the symmetry of the crystal. The independent values are the values defined on the coordinates within the asymmetric part of the unit cell. For all space groups, the asymmetric unit is known (Hahn, 1995), but an automated procedure does not exist for generating the asymmetric unit for space groups of arbitrary dimensions (Engel, 1986). However, it is much easier to derive the symmetry properties of the discretized density and the (discrete) structure factors. The independent density values $\rho_{j}^{\text {au }} \quad\left(j=1, \ldots, N_{\text {pix }}^{\text {au }}\right)$ are determined by consecutively considering all the points $\mathbf{x}_{k}$ and selecting only those values for

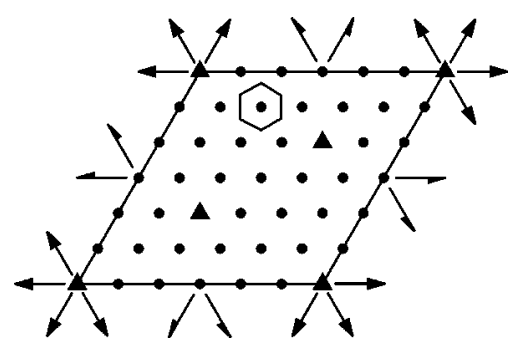

Figure 1

Base plane of a hexagonal unit cell with a symmetry-adapted grid of $N_{1}=N_{2}=6$. The symmetry elements of the space group $P 321$ as well as the Wigner-Seitz shape of one voxel are indicated. which $\mathbf{x}_{k}$ is not equivalent by symmetry to a previously selected value, i.e. we arbitrarily select from each orbit the $\mathbf{x}_{k}$ with the lowest $k$. Within the same procedure, a table is generated that stores for each independent $\rho_{j}^{\text {au }}$ the $k$ values of all points in its orbit as well as its multiplicity $m_{j}^{\rho}$ (the $\rho$ table). This table completely defines the symmetry of $\left\{\rho_{k}\right\}$. It can be used to expand the unique values $\left\{\rho_{j}^{\text {au }}\right\}$ into the density of the entire unit cell, and to extract the unique values from the density $\left\{\rho_{k}\right\}$ of the unit cell. In a similar procedure, a table is made that defines all the points $k$ corresponding to the unique structure factors $F(\mathbf{H})$ (the $F$ table).

The symmetry has consequences for the choice of the grid of the discrete density. The pixels must be chosen such that each symmetry operator transforms a pixel onto itself or onto another pixel. This implies that the voxels (the space around each pixel) must have the shape of the Wigner-Seitz unit cell of the lattice (Schneider \& van Smaalen, 2000).

Secondly, symmetry puts severe restrictions on the divisions along the axes, i.e. on the numbers $N_{j}$ [equation (2)]. Because symmetry elements, like twofold axes and mirror planes, are repeated half-way between the lattice points, $N_{j}$ must be an even integer for almost all (super-)space groups. If higherorder rotation axes are present, further restrictions apply. For example, in hexagonal space groups, $N_{j}$ along directions perpendicular to the unique axis must be a threefold integer. If a $6_{1}$ screw axis is present, the division along the direction of the axis must be a sixfold integer. If directions in the lattice are equivalent by symmetry, the divisions along these directions must be equal. These restrictions lead to the notion that a symmetry-adapted grid should be used for the definition of the electron-density function (Fig. 1) (Schneider \& van Smaalen, 2000).

Symmetry restricts the choice of the origin of the grid. Either symmetry elements coincide with grid points or symmetry elements coincide with the boundaries of the voxels (which are in between grid points) (Fig. 2). We believe that the better choice is that the grid points are chosen on the symmetry elements. In this case, each grid point has a multiplicity $m_{j}^{\rho}$ assigned to it, which counts the number of equivalent grid points in the unit cell. The second choice of the origin would result in artifacts represented by rows or plains of pairs

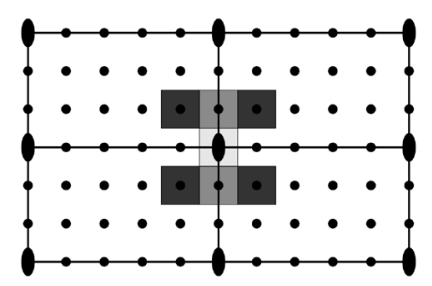

(a)

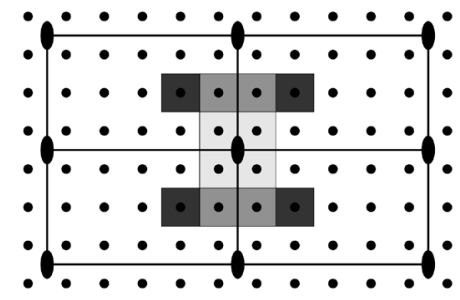

(b)
Figure 2

Unit cell of a two-dimensional rectangular lattice with a symmetryadapted grid of $N_{1}=6$ and $N_{2}=10$. (a) More favourable, and (b) less favourable choices of the positions of the grid points defining $\rho_{k}$ [equation (3)]. The symmetry elements of the space group $p 2 \mathrm{~mm}$ are indicated. Voxels (pixels) that are equivalent by symmetry are indicated by the same shading. 
Table 1

Experimental data and results for the electron density of $\mathrm{NaV}_{2} \mathrm{O}_{5}$ at room temperature.

\begin{tabular}{ll}
\hline Chemical formula & $\mathrm{NaV}_{2} \mathrm{O}_{5}$ \\
Chemical formula weight & 172.87 \\
Space group & $P m m n$ \\
Lattice parameters $a, b, c(\AA)$ & $11.3113(1), 3.6098(1), 4.8018(1)$ \\
Volume $\left(\AA^{3}\right)$ & $196.065(5)$ \\
$Z$ & 2 \\
Radiation type & Mo $K \alpha$ \\
Wavelength $(\AA)$ & 0.7107 \\
Absorption coefficient $\left(\mathrm{mm}^{-1}\right)$ & 4.706 \\
Crystal size $\left(\mathrm{mm}^{3}\right)$ & $0.1 \times 0.1 \times 0.1$ \\
Crystal colour & Colourless \\
Diffractometer & Nonius MACH3 \\
Detector & Scintillation \\
Data-collection method & $\omega-2 \theta$ scans \\
Absorption correction & $\psi$ scans \\
No. of measured reflections & 4342 \\
No of independent reflections & 1158 \\
No. of observed reflections & 2979 \\
No. of independent observed reflections & 930 \\
Criterion for observed reflections & $I>3.0 \sigma(I)$ \\
$R_{\text {int }}($ all reflections) & 0.023 \\
$R_{\text {int }}$ (observed reflections) & 0.020 \\
Maximum of sin $(\theta) / \lambda\left(\AA^{-1}\right)$ & 1.08 \\
Range of $h, k, l$ & $-24 \rightarrow h \rightarrow 24$ \\
& $0 \rightarrow k \rightarrow 7$ \\
Refinement on & $-10 \rightarrow l \rightarrow 10$ \\
Weighting scheme & $F$ \\
$R$ (observed reflections) & {$\left[\sigma^{2}(F)+(0.009 F)^{2}\right]^{-1}$} \\
$w R$ (observed reflections) & 0.023 \\
$R$ (all reflections) & 0.025 \\
$w R$ (all reflections) & 0.037 \\
Goodness of fit (obs., all) & 0.026 \\
No. of parameters & $1.40,1.30$ \\
Extinction correction & 28 \\
& $\mathrm{Isotropic} \mathrm{type} \mathrm{I}$ \\
Extinction coefficient & $(\mathrm{Becker} \mathrm{\&} \mathrm{Coppens,} \mathrm{1974)}$ \\
Source of atomic scattering factors & $0.065(4)$ \\
\hline & $\mathrm{Su} \mathrm{\&} \mathrm{Coppens}(1997)$ \\
\hline &
\end{tabular}

of points with equal densities (Fig. 2). Then each grid point would be at a general position.

\subsection{Symmetry and the Fourier transform}

The structure factors $F_{\text {MEM }}(\mathbf{H})$ are computed from the electron density $\left\{\rho_{k}\right\}$ by a discrete Fourier transform, employing a generalization of the Beevers-Lipson algorithm in combination with a 1D fast Fourier transform (FFT) (Schneider \& van Smaalen, 2000). With the exception of some symmetry operators in 2D and 3D space, it is not possible to incorporate the effects of symmetry into the Beevers-Lipson and FFT algorithms. Therefore, we have designed BayMEM to always compute the Fourier transforms for the complete unit cell. This requires storage of $2 N_{\text {pix }}$ real numbers. The computational complexity was shown to be proportional to $N_{\text {pix }} \log \left(N_{\text {pix }}\right)$ (Schneider \& van Smaalen, 2000).

In the Fourier transform, symmetry is handled by expanding the independent density values $\left\{\rho_{j}^{\mathrm{au}}\right\}$ using the $\rho$ table, computing the Fourier transform, and finally extracting the unique structure factors $F(\mathbf{H})$ using the $F$ table. All other Fourier transforms are handled in a similar way, using the $\rho$ table in direct space and the $F$ table for the reflections. Other computations directly use the stored unique density values $\left\{\rho_{j}^{\mathrm{au}}\right\}$ and the observed unique reflections $F(\mathbf{H})$.

\subsection{Symmetry and the entropy}

Continuity requires that the entropy of a map with symmetry is again defined by (4). With respect to the unique density values $\left\{\rho_{j}^{a u}\right\}$, this transforms into

$$
S=-\sum_{j=1}^{N_{\mathrm{pix}}^{\mathrm{au}}} m_{j}^{\rho} \rho_{j}^{\mathrm{au}} \log \left(\rho_{j}^{\mathrm{au}} / \tau_{j}^{\mathrm{au}}\right) .
$$

This definition is opposite to the definition of the $F$ constraint, which usually pertains to a sum over the unique reflections only [equation (5)]. It results in the modified formal solution of the MaxEnt equations, given by

$$
\rho_{j}^{\mathrm{au}}=\tau_{j}^{\mathrm{au}} \exp \left[-\lambda \frac{1}{m_{j}^{\rho}} \frac{\partial C_{F}}{\partial \rho_{j}^{a u}}\right] .
$$

The occurrence of $m_{j}^{\rho}$ in (9) cancels against the occurrence of $m_{j}^{\rho}$ in the expression of the structure factor, resulting in

$$
\begin{aligned}
\frac{1}{m_{j}^{\rho}} \frac{\partial C_{F}}{\partial \rho_{j}^{a u}}= & \sum_{i=1}^{N_{F}} \frac{1}{\sigma^{i}(\mathbf{H})^{2}}\left[F_{\text {obs }}^{i}(\mathbf{H})-F_{\mathrm{MEM}}^{i}(\mathbf{H})\right] \\
& \times \exp \left[2 \pi i \mathbf{H}^{i} \cdot \mathbf{x}_{j}\right]+\text { c.c. },
\end{aligned}
$$

where c.c. indicates complex conjugate.

\section{The computer program BayMEM}

Based on the considerations in $\$ 2$, a computer program BayMEM was written for the application of the MEM to aperiodic crystals. Both the Sakata-Sato algorithm (Sakata \& Sato, 1990) and the Cambridge algorithm (Skilling \& Bryan, 1984) can be used. New computer code was written for the Sakata-Sato algorithm, whereas the Cambridge algorithm was made available by incorporating the set of subroutines MEMSYS5 v. 1.2 (Gull \& Skilling, 1999a) into BayMEM. It is noticed that BayMEM contains a special algorithm for the $n \mathrm{D}$ fast Fourier transform (Schneider \& van Smaalen, 2000), and that it contains a newly developed algorithm for handling the symmetry in arbitrary dimensions (\$\$2.2-2.4). The two MaxEnt algorithms solely pertain to how equation (9) is iteratively solved for variation of the unique pixels.

\subsection{Periodic crystals}

Electron densities of periodic crystals in three-dimensional space can be computed with BayMEM too $(d=0)$. We have tested BayMEM for the case of silicon, using the accurate structure factors of $\mathrm{Si}$ as measured by Saka \& Kato (1986). The resulting electron-density maps are indistinguishable from those obtained with the computer program $M E E D$ (Kumazawa et al., 1993), and we have observed the same features and artifacts as have been extensively discussed in the literature (de Vries et al., 1996; Takata \& Sakata, 1996).

As a second test case, we have computed the electron density of $\mathrm{NaV}_{2} \mathrm{O}_{5}$, using X-ray intensity data measured up to 
Table 2

Results and computational details for the MEM applied to $\mathrm{NaV}_{2} \mathrm{O}_{5}$.

\begin{tabular}{llll}
\hline & $\begin{array}{l}\text { BayMEM: } \\
\text { Cambridge algorithm }\end{array}$ & $\begin{array}{l}\text { BayMEM: } \\
\text { Sakata-Sato algorithm }\end{array}$ & MEED \\
\hline$N_{\text {pix }}$ & 524288 & & \\
$N_{\text {pui }}^{\text {au }}$ & 68641 & & \\
$N_{\text {ref }}$ & 1158 & 25 & 1220 \\
RAM (Mbyte) & 25 & 12.4 & 275.0 \\
CPU (min) & 6.7 & 0.0282 & 0.0282 \\
$R$ & 0.0248 & 0.0205 & 0.0205 \\
$w R$ & 0.0206 & & \\
\hline
\end{tabular}

high angles (Table 1). Structure refinements were performed with JANA98 (Petricek \& Dusek, 1998), using atomic form factors for spherical atoms and anisotropic temperature factors. The atomic coordinates published in Smolinski et al. (1998) were confirmed. MaxEnt reconstructions of the electron density were computed from the observed structurefactor amplitudes together with the phases of the calculated structure factors of the final refinement. The electron density was discretized on a grid of $128 \times 64 \times 64$ pixels.

Separate runs of BayMEM were made using the Cambridge and Sakata-Sato algorithms. A third computation was performed with the MEED program (Kumazawa et al., 1993). The calculations were considered to be converged when the $F$
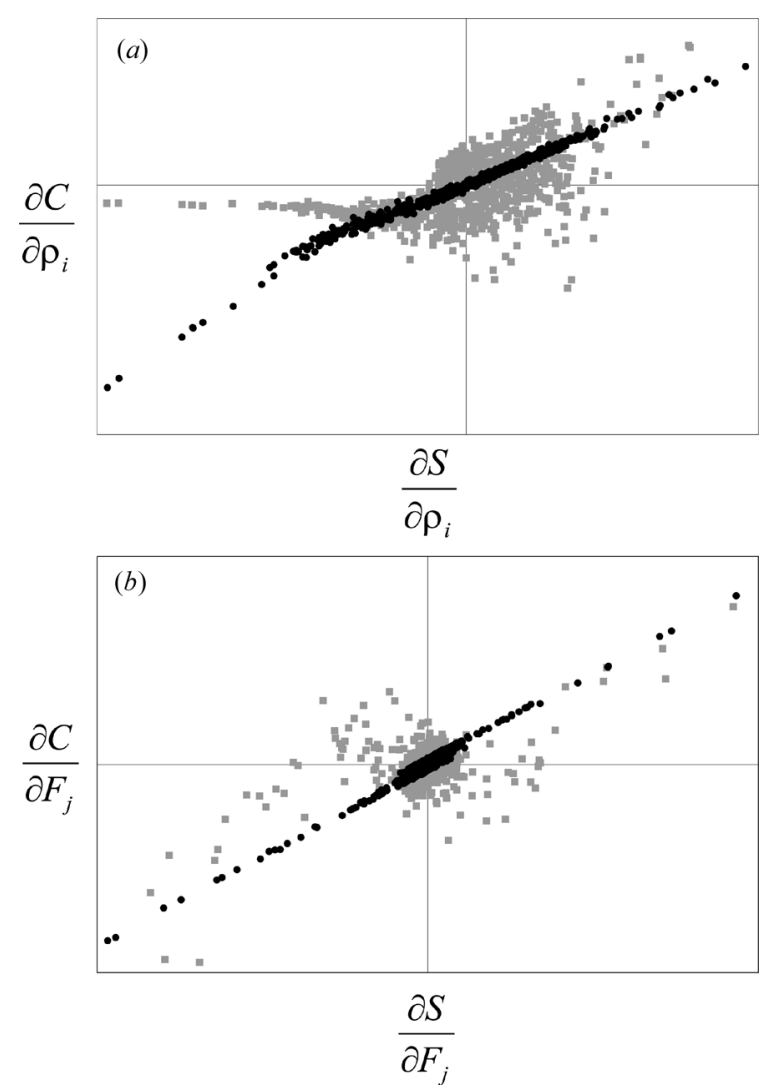

Figure 3

Graphical representation of $(a)$ equation (11), and $(b)$ equation (12). Grey squares: Sakata-Sato algorithm. Black circles: Cambridge algorithm. Only about $1 \%$ of all points shown in $(a)$. For an ideal MaxEnt solution, all points lie on a straight line. constraint was fulfilled (Table 2). The resulting electron densities $\rho^{\text {MEM }}$ were similar to each other, with the differences between them less than the apparent noise in the individual maps. The electron density of $\mathrm{NaV}_{2} \mathrm{O}_{5}$ is well reproduced by $\rho^{\mathrm{MEM}}$.

Our results for $\mathrm{Si}$ and $\mathrm{NaV}_{2} \mathrm{O}_{5}$ show that BayMEM and $M E E D$ give the same result for the reconstructed electron density in the case of periodic crystals. A second point of comparison is the computational efficiency of the two computer programs. Table 2 shows that BayMEM needs much less RAM and is much faster than $M E E D$ for the case of $\mathrm{NaV}_{2} \mathrm{O}_{5}$. This result reflects the efficiency of FFT algorithms (Schneider \& van Smaalen, 2000). For problems of smaller sizes (less pixels and less reflections), the differences between the two programs become smaller and, in the case of silicon (1649 unique pixels and 30 reflections), MEED is even faster than BayMEM (Schneider, 2001). However, MaxEnt computations for larger structures require the use of FFT algorithms combined with an efficient handling of symmetry, as is implemented into BayMEM.

\subsection{Algorithms}

BayMEM works with both the Cambridge and the SakataSato algorithms. Thus the performance of the two algorithms can be compared under otherwise identical conditions. To be able to assess the quality of the MaxEnt reconstructions, we have used simulated noisy data of oxalic acid dihydrate that were obtained from calculated structure factors of a model electron density [for details see Palatinus \& van Smaalen (2002)].

For the optimum electron density $\rho^{\mathrm{MEM}}$, the entropy and constraint should fulfil the following set of equations:

$$
\frac{\partial S}{\partial \rho_{i}}=\lambda \frac{\partial C}{\partial \rho_{i}}
$$

for $i=1, \ldots, N_{\text {pix }}^{\mathrm{au}}$. Alternatively, they should fulfil the equivalent set of equations in reciprocal space:

$$
\frac{\partial S}{\partial F_{j}}=\lambda \frac{\partial C}{\partial F_{j}}
$$

for $j=1, \ldots, N_{F}$.

The Cambridge algorithm is supposed to produce an electron density that is close to the real MaxEnt solution because $\rho$ and $\lambda$ are optimized simultaneously. On the other hand, there is no a priori reason to expect that the Sakata-Sato algorithm will produce an electron density that fulfils (11) and (12), because the Sakata-Sato algorithm uses an estimated value for $\lambda$ and it determines $\rho^{\mathrm{MEM}}$ by an approximate iterative procedure. The numerical evaluation for the case of oxalic acid dihydrate confirms these expectations. The electron density produced by the Cambridge algorithm is relatively close to the perfect solution, while the Sakata-Sato algorithm produces distributions far from the optimum (Fig. 3).

Of practical importance is to know how close the optimized electron density $\rho^{\mathrm{MEM}}$ is to the true electron density $\rho^{\text {true }}$. The latter is known for the simulated data that were used here. For 
the Sakata-Sato algorithm, it was shown in a previous publication that $\Delta \rho_{\text {Sakata }}=\rho^{\mathrm{MEM}}($ Sakata - Sato $)-\rho^{\text {true }}$ is small in the case of a procrystal prior while it has variations up to a few electrons per $\AA^{3}$ in the case of a flat prior (Palatinus \& van Smaalen, 2002). Here we will directly compare the optimized electron densities of the Sakata-Sato and Cambridge algorithms, employing the quantity

$$
\frac{\left|\Delta \rho_{\text {Cambridge }}\right|}{\left|\Delta \rho_{\text {Cambridge }}\right|+\left|\Delta \rho_{\text {Sakata }}\right|},
$$

where $\Delta \rho_{\text {Cambridge }}$ is defined analogously to $\Delta \rho_{\text {Sakata }}$. A value less than 0.5 indicates a point where the Cambridge algorithm was better, while values larger than 0.5 indicate points where the Sakata-Sato algorithm was better. Depending on the resolution of the map and the noise level of the data, average values of (13) were found to lie between 0.46 and 0.47 in the case of a flat prior and between 0.495 and 0.499 in the case of a procrystal prior. These values show that on the average the Cambridge algorithm produces a slightly better density than the Sakata-Sato algorithm. However, none of the algorithms is clearly superior and the variations of the values of (13) over the individual points show that there are regions where one or the other algorithm performs better (Fig. 4).

The third criterion for comparison is the speed of convergence of the algorithms. In our tests, the Cambridge algorithm was usually faster than the Sakata-Sato algorithm if the classical $F_{2}$ constraint was used. A serious problem with the Sakata-Sato algorithm is that the convergence sometimes effectively stops before the constraint is fulfilled. This happens especially for large problems and cases where the standard uncertainties have been underestimated.

Because the Cambridge algorithm produces an electron density that is closer to the true electron density than the Sakata-Sato algorithm, and because it converges faster and more reliably, its use is recommended if the $F_{2}$ constraint is used. However, the Cambridge algorithm does not allow use of any other constraint than the $F_{2}$ constraint. The $F_{n}$ constraint with $n$ equal to 4 or 6 was shown to converge significantly faster and to lead to better results than the $F_{2}$ constraint, especially in the medium- and low-density regions (Palatinus \& van Smaalen, 2002). This improvement turns out to be larger than the difference between the electron densities obtained with the Cambridge and Sakata-Sato algorithms. Thus, if the fine features of the electron density are investigated, the use of the Sakata-Sato algorithm with $F_{4}$ or $F_{6}$ constraint is preferred.

Finally, it is noticed that a truly Bayesian analysis corresponds to a stopping criterion other than the fulfilment of the $F_{2}$ constraint. This leads to convergence beyond the point $C_{F}=0$ (Gull \& Skilling, 1999b). This approach is implemented in the MEMSYS5 package and has been tested, too. Our results show that using this 'true Bayesian' maximum entropy leads to strong overfitting of the data and consequently to much noisier density maps than the classical constraint. We conclude that this approach in its present form is not suitable for crystallographic MaxEnt applications.

\section{The inorganic misfit layer compound (LaS) ${ }_{1.14} \mathrm{NbS}_{2}$}

Inorganic misfit layer compounds belong to the class of incommensurate composite crystals (van Smaalen, 1995; Wiegers, 1996). We have performed MaxEnt calculations on the two isostructural misfit layer compounds $(\mathrm{LaS})_{1.14} \mathrm{NbS}_{2}$ and $(\mathrm{LaS})_{1.13} \mathrm{TaS}_{2}$, employing the computer program BayMEM. The results for both compounds turned out to be
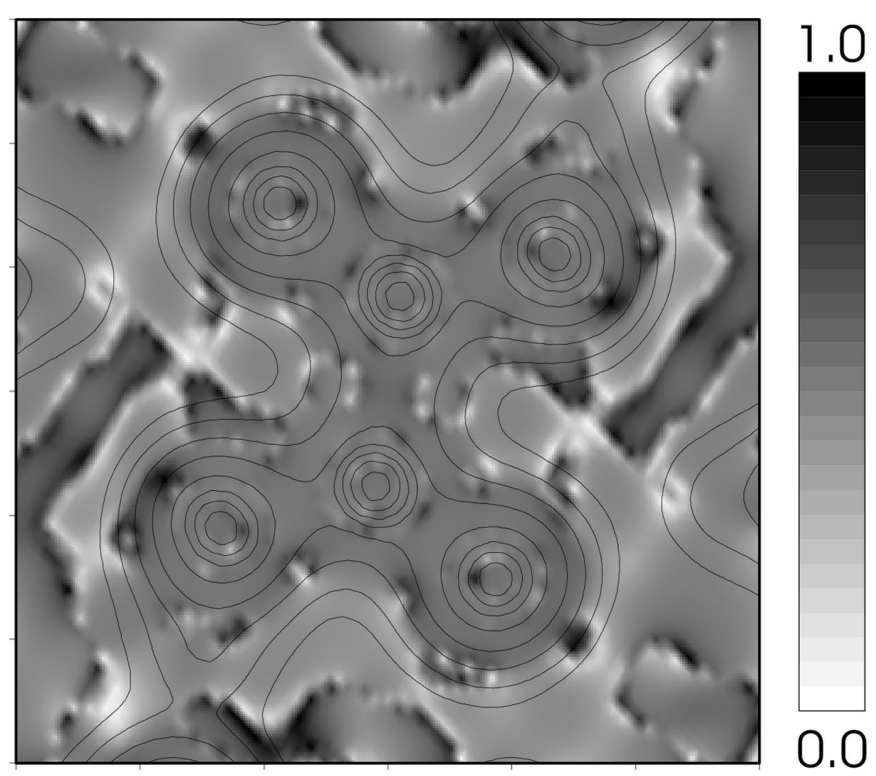

(a)

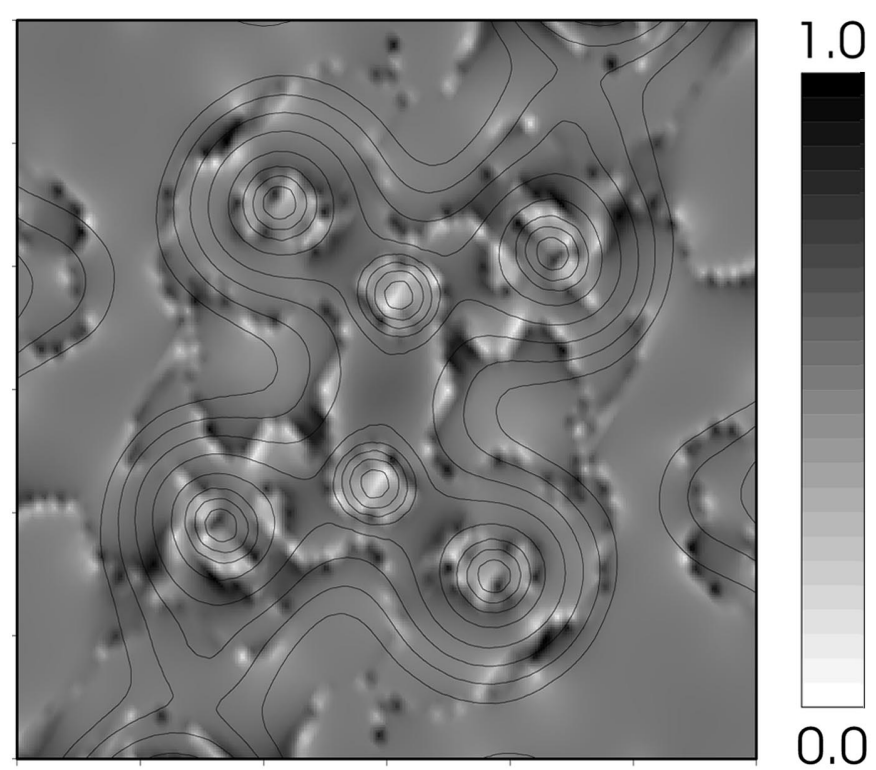

(b)

Figure 4

Comparison of the electron densities obtained with the Sakata-Sato and Cambridge algorithms. (a) For a flat prior, and $(b)$ for a procrystal prior. A section through the plane of the oxalic acid molecule is shown. Contour lines at intervals $2^{n}, n=-2, \ldots, 5$, indicate $\rho^{\text {true }}$. The values of equation (13) are represented on a greyscale. Light tones mark areas where the Cambridge algorithm produces better density values and dark tones mark areas where the Sakata-Sato algorithm produces better values. Computations were performed with BayMEM employing the data set n1r1.00 (for details, see Palatinus \& van Smaalen, 2002). 
Table 3

Computational details and results for the MEM calculations on $\mathrm{LaS}_{114} \mathrm{NbS}_{2}$.

\begin{tabular}{|c|c|c|c|c|}
\hline & \multicolumn{2}{|c|}{ Observed data } & \multicolumn{2}{|c|}{ Calculated data } \\
\hline & $\begin{array}{l}\text { Cambridge } \\
\text { algorithm }\end{array}$ & $\begin{array}{l}\text { Sakata-Sato } \\
\text { algorithm }\end{array}$ & $\begin{array}{l}\text { Cambridge } \\
\text { algorithm }\end{array}$ & $\begin{array}{l}\text { Sakata-Sato } \\
\text { algorithm }\end{array}$ \\
\hline No. of reflections & 10237 & & & \\
\hline$N_{\text {pix }}$ & 16777216 & & & \\
\hline$N_{\text {pix }}^{\text {pau }}$ & 1052736 & & & \\
\hline RAM (Mbyte) & 678 & & & \\
\hline Computation time (h) & 76.8 & 73.9 & 11.4 & 4.4 \\
\hline Constraint [equation (6)] & $F_{2}$ & $F_{4}$ & $F_{2}$ & $F_{4}$ \\
\hline Final value of constraint & 1.0 & 58.4 & 1.0 & 1.0 \\
\hline \multicolumn{5}{|l|}{$R$ values obs./all (\%): } \\
\hline All reflections & $2.1 / 4.1$ & $2.8 / 6.4$ & $3.2 / 3.1$ & $2.7 / 3.4$ \\
\hline Main reflections & $1.6 / 1.7$ & $2.2 / 2.3$ & $3.9 / 3.9$ & $2.7 / 2.8$ \\
\hline First-order satellites & $3.7 / 8.6$ & $4.8 / 14.1$ & $0.6 / 0.8$ & $2.4 / 3.4$ \\
\hline Second-order satellites & $3.8 / 16.8$ & $6.2 / 28.7$ & $0.6 / 1.6$ & $3.3 / 8.7$ \\
\hline
\end{tabular}

satellites are observed. The model included the Fourier components up to second harmonics for the modulation functions for the displacements and the temperature factors. Furthermore, the average occupation of La was refined towards 0.949 (2), and the modulation function for this occupancy was included in the model.

The phases of the reflections were taken from the calculated structure factors of the final structure model [model D in Jobst \& van Smaalen (2002)]. Together with the observed structure-factor amplitudes, they formed the observed data (subscript obs) that were used in the MaxEnt calculations. Standard uncertainties are based on counting statistics. The scaling towards the scattering of the unit cell and the corrections for the anomalous scattering were similar in every way. Therefore, we present here in detail only the results for $(\mathrm{LaS})_{1.14} \mathrm{NbS}_{2}$.

\subsection{The structure model and experimental data}

The structure has been solved and refined using conventional crystallographic methods (van Smaalen, 1991; Jobst \& van Smaalen, 2002). The data and model published in Jobst \& van Smaalen (2002) were used in the present work, and we refer to Jobst \& van Smaalen (2002) for experimental details and structural parameters (Fig. 5).

Important for the present analysis is that a complete data set is available up to $\sin (\theta) / \lambda=1.01 \AA^{-1}$. Almost all $(98 \%)$ of the main reflections are of the type observed [they have $I>3 \sigma(I)]$, whereas about half of the first- and second-order

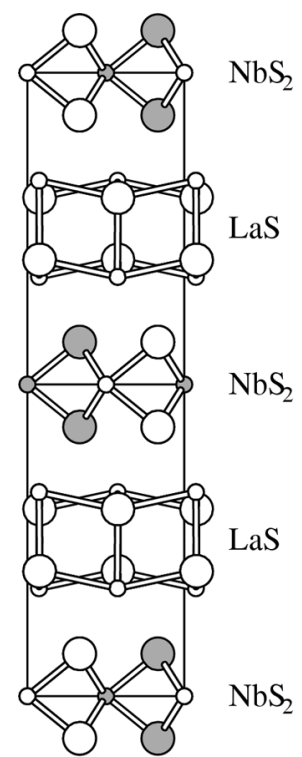

(a)

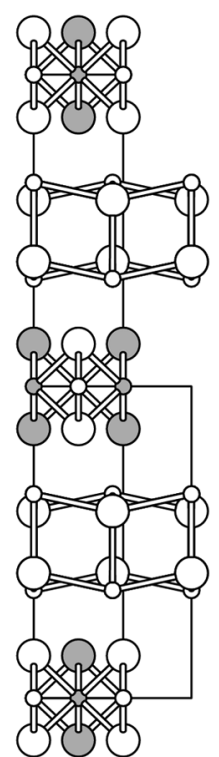

(b)
Figure 5

The average structure of $(\mathrm{LaS})_{1.14} \mathrm{NbS}_{2}$. (a) Projection along the incommensurate $a$ axis. (b) Projection along the common $b$ axis. Large circles denote $\mathrm{S}$ atoms, small circles represent the metal atoms. Shaded and white circles represent atoms at different positions of the projected coordinate. Lattice constants at $T=115 \mathrm{~K}$ are: $a_{1}=3.3065, a_{2}=5.7983$, $b=5.7960$ and $c=22.957 \AA$. obtained by a procedure described elsewhere (Bagautdinov $e t$ al., 1998).

A second data set was formed by the structure factors computed for the final structure model, albeit without the contributions of the anomalous scattering factors. They were denoted as calculated data (subscript calc). To be able to apply the MaxEnt procedure, non-zero standard uncertainties must be assigned to each reflection. Standard uncertainties of the calculated data were set equal to the standard uncertainties of the observed data. The calculated data correspond to the Fourier transform of the model electron density. Their use as 'observed' data in the MEM [equation (6)] thus allows one to quantitatively estimate the quality of the MaxEnt reconstruction of the electron density for a model that is as close as possible to the real electron density.

\subsection{Details of the computations}

The electron density was calculated on a grid of $32 \times 64 \times 256 \times 32$ pixels. This corresponded to a resolution

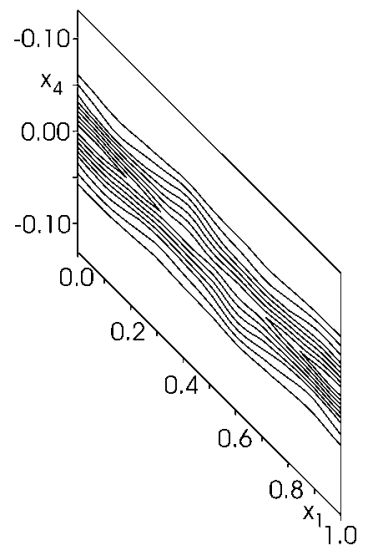

(a)

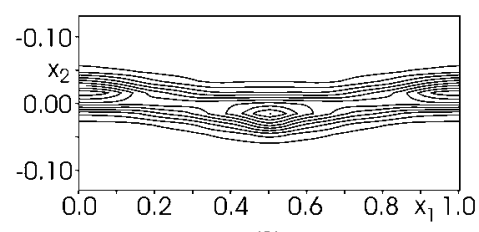

(b)

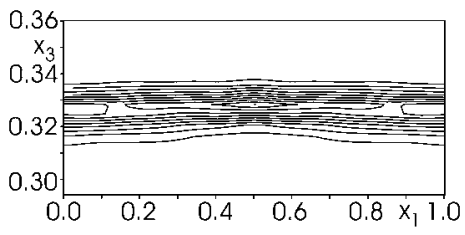

(c)
Figure 6

Sections of the electron density $\rho_{\mathrm{obs}}^{\mathrm{MEM}}$ at the position of La. (a) $x 1-x 4$ section with $\rho_{\max }=961.1 \mathrm{e}^{-4} ; \quad(b) \quad x 2-x 4$ section with $\rho_{\max }=1409.9 \mathrm{e}^{-4} ; \quad(c) \quad x 3-x 4$ section with $\rho_{\max }=1161.4 \mathrm{e} \AA^{-4}$. Contour lines are shown at intervals of $10 \%$ of the maximum value $\rho_{\max }$ of the electron density in the corresponding sections. 
of $0.103 \times 0.092 \times 0.090 \times 0.181 \AA$. A finer grid might be desirable but then the calculations would have been too time consuming. A flat prior was used throughout the whole analysis.

$\rho_{\mathrm{obs}}^{\mathrm{MEM}}$ was obtained from a run of BayMEM using the observed data and the Cambridge algorithm (Fig. 6). In a similar way, $\rho_{\text {calc }}^{\mathrm{MEM}}$ was obtained from the calculated data. Calculations with the Sakata-Sato algorithm and the $F_{4}$ constraint did not converge within a reasonable time (see §3.2) and the iteration had to be stopped before the $F_{4}$ criterion was fulfilled. Therefore, we have only used the results obtained with the Cambridge algorithm in the present analysis. Details of the MaxEnt computations are summarized in Table 3.

BayMEM can save the electron densities in several formats. An internal format is used to store all independent density values in the full double precision that is necessary to maintain the accuracy of the calculations. The computer program JANA2000 (Petricek \& Dusek, 2000) is used for the visualization of the electron density and BayMEM can save the electron density in a format suitable for JANA2000.
A principal task of the analysis of the $\rho^{\mathrm{MEM}}$ is to extract the modulation functions for the displacive modulation of the atoms from it. This involves:

(a) The computation of the electron densities in 3D sections of superspace from $\rho^{\mathrm{MEM}}$ for a series of $t$ values ( $t$ maps), each of them representing the electron density in physical space.

(b) Determination of the maxima in each $t$ map. The maxima of the electron density in physical space are then identified with the atomic positions in this particular section. For each step, it is necessary to be able to compute the electron density for arbitrary values of the coordinates. Because $\rho^{\mathrm{MEM}}$ is defined on a grid only, an interpolation method is required. We have used the bicubic spline method (Press et al., 1996), generalized to arbitrary dimensions.

Using this two-step procedure, it is possible to extract the positions of the individual atoms from the $\rho^{\mathrm{MEM}}$ as a function of the parameter $t$ with arbitrary dense sampling in $t$. The difference between the modulated and the average positions then defines the modulation function. For all four crystallographically independent atoms of $(\mathrm{LaS})_{1.14} \mathrm{NbS}_{2}$, we have extracted the modulation functions from $\rho_{\text {calc }}^{\mathrm{MEM}}$ and $\rho_{\mathrm{obs}}^{\mathrm{MEM}}$
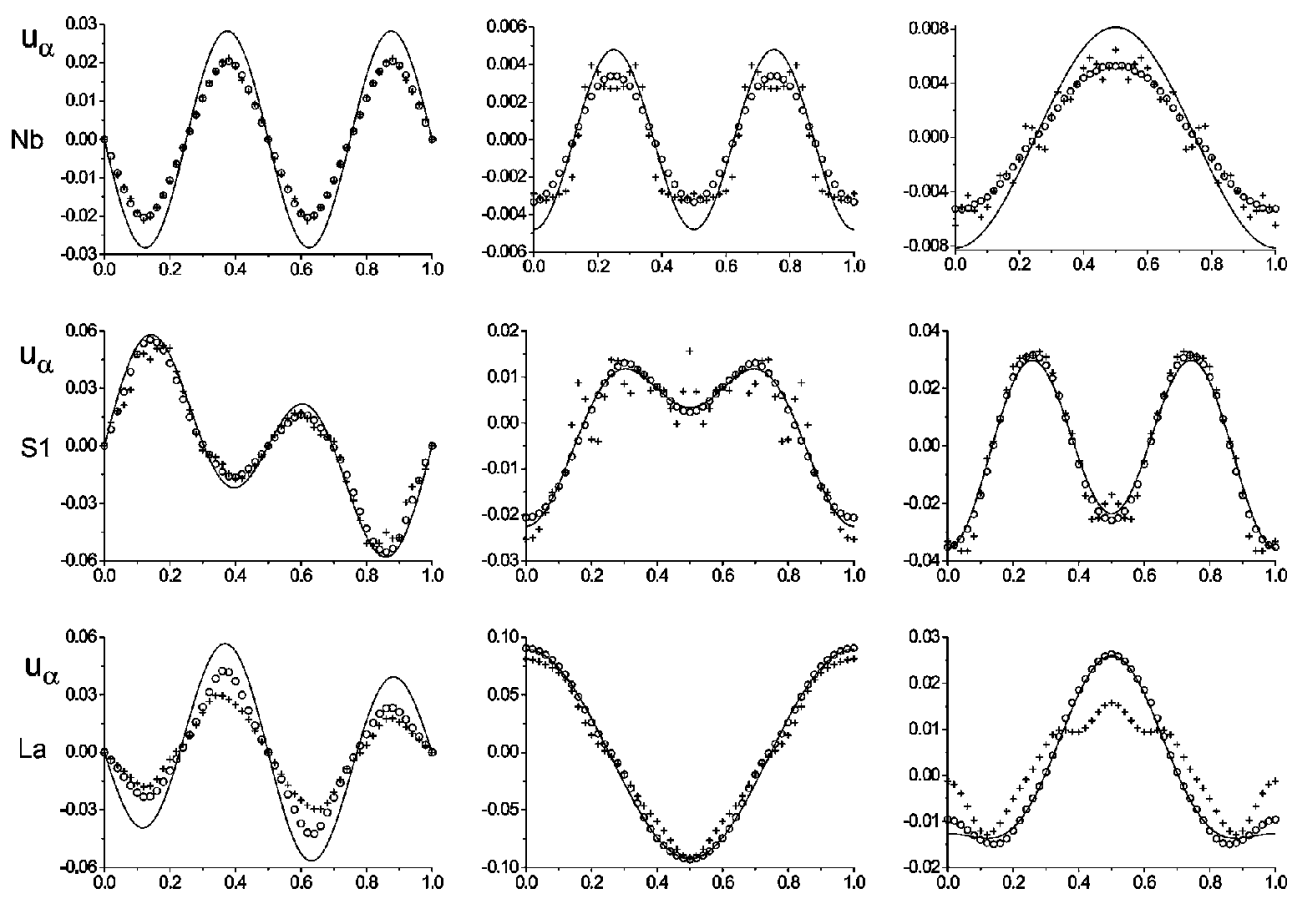

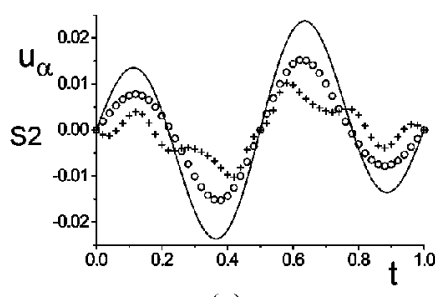

(a)

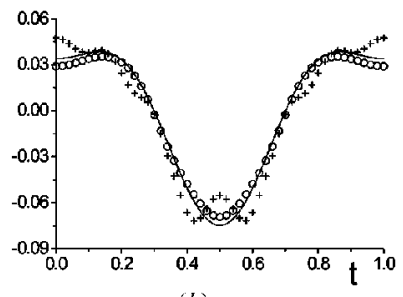

(b)

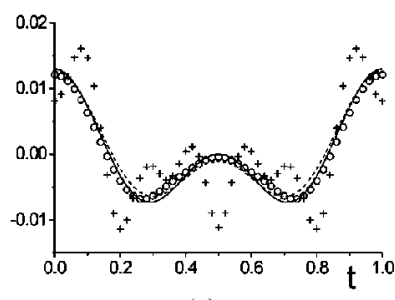

(c)

Figure 7

Overview of the modulation functions of the independent atoms of $\mathrm{LaS}_{1.14} \mathrm{NbS}_{2}$. Full lines: model modulation functions; open circles: modulation extracted from $\rho_{\text {calc }}^{\mathrm{MEM}}$; crosses: modulation extracted from $\rho_{\mathrm{obs}}^{\mathrm{MEM}}$; dashed line for $u_{c}$ of S2: best harmonic fit to the $\rho_{\mathrm{obs}}^{\mathrm{MEM}}$. Horizontal scale: $t$, vertical scale: deviation from the average position along the respective directions $a, b$ and $c$ in $\AA$. 


\section{Table 4}

The difference between the modulation functions extracted from $\rho_{\text {calc }}^{\text {MEM }}$ and those contained in the model.

Given is the value of the difference averaged over $t$, both in units of length $(\AA)$ and as fraction of the pixel size (\%). Only values for the modulation along the $a$ axis are given. $\rho_{\text {calc }}^{\text {MEM }}$ was obtained with a grid of $64 \times 64 \times 256 \times 64$ pixels. The values are given for both the Cambridge and Sakata-Sato algorithms.

\begin{tabular}{llllll}
\hline & \multicolumn{2}{l}{ Cambridge algorithm } & & \multicolumn{2}{l}{ Sakata-Sato algorithm } \\
\cline { 2 - 3 } Atom & $\AA$ & & & $\AA$ & $\%$ \\
\hline $\mathrm{Nb}$ & 0.0010 & 2.1 & & 0.0028 & 5.6 \\
$\mathrm{~S} 1$ & 0.0017 & 3.3 & & 0.0023 & 4.7 \\
$\mathrm{La}$ & 0.0026 & 2.9 & & 0.0027 & 3.0 \\
$\mathrm{~S} 2$ & 0.0010 & 1.1 & & 0.0016 & 1.8 \\
\hline
\end{tabular}

Table 5

The difference between the modulation functions extracted from $\rho_{\text {calc }}^{\mathrm{MEM}}$ and those contained in the model.

Given is the value of the difference averaged over $t$, both in units of length $(\AA)$ and as fraction of the pixel size (\%). The values are given for $\rho_{\text {calc }}^{\mathrm{MEM}}$ obtained with the Cambridge algorithm and for $\rho_{\text {calc }}^{\mathrm{MEM}}$ obtained with the Sakata-Sato algorithm. Note that both algorithms lead to results of comparable quality.

\begin{tabular}{|c|c|c|c|c|c|}
\hline \multirow[b]{2}{*}{ Atom } & \multirow[b]{2}{*}{ Axis } & \multicolumn{2}{|c|}{ Cambridge algorithm } & \multicolumn{2}{|c|}{ Sakata-Sato algorithm } \\
\hline & & $\bar{\AA}$ & $\%$ & $\bar{\AA}$ & $\%$ \\
\hline \multirow[t]{3}{*}{$\mathrm{Nb}$} & $a$ & 0.0057 & 5.6 & 0.0065 & 6.4 \\
\hline & $b$ & 0.0028 & 3.0 & 0.0019 & 2.1 \\
\hline & $c$ & 0.0018 & 2.0 & 0.0023 & 2.5 \\
\hline \multirow[t]{3}{*}{ S1 } & $a$ & 0.0043 & 4.2 & 0.0041 & 3.9 \\
\hline & $b$ & 0.0024 & 2.6 & 0.0023 & 2.5 \\
\hline & $c$ & 0.0018 & 2.0 & 0.0019 & 2.2 \\
\hline \multirow[t]{3}{*}{$\mathrm{La}$} & $a$ & 0.0120 & 6.7 & 0.0114 & 6.3 \\
\hline & $b$ & 0.0020 & 2.2 & 0.0012 & 1.3 \\
\hline & $c$ & 0.0009 & 1.0 & 0.0015 & 1.7 \\
\hline \multirow[t]{3}{*}{ S2 } & $a$ & 0.0049 & 2.7 & 0.0046 & 2.5 \\
\hline & $b$ & 0.0025 & 2.8 & 0.0023 & 2.5 \\
\hline & $c$ & 0.0031 & 3.5 & 0.0023 & 2.5 \\
\hline
\end{tabular}

accordingly, employing 50 equally spaced points on the interval $0 \leq t<1$ (Fig. 7).

\subsection{Discussion}

With the calculated data as 'observed' data [equation (5)], BayMEM should reproduce the electron density of the model that was used to generate the calculated structure factors. Fig. 7 shows that the modulation functions that are determined from $\rho_{\text {calc }}^{\mathrm{MEM}}$ indeed follow the modulation functions of the structure model quite well. For some modulation functions, the match is almost perfect (e.g. the modulation of La along $y$ ), while for other modulation functions differences between the model and $\rho_{\text {calc }}^{\mathrm{MEM}}$ are found (e.g. the modulation of $\mathrm{Nb}$ along $x$ ).

A number of reasons exist why $\rho_{\text {calc }}^{\mathrm{MEM}}$ will not reproduce the electron density of the model exactly (see below). However, the major source of the difference between the reconstructed modulation functions and the model is the finite resolution of the grid that is used to define the electron density in the MaxEnt calculations. First of all, it is noted that the sizes of the modulation functions are of the same order as the grid size of about $0.1 \AA$. It then becomes apparent that the differences between the model and the modulation functions extracted from $\rho_{\text {calc }}^{\mathrm{MEM}}$ are only a few percent of the pixel size, with the largest deviation being less than $10 \%$ of the pixel size. Indeed, it cannot be expected to obtain a more accurate estimate of the positions of the maxima in $\rho^{\mathrm{MEM}}$ than a few percent of the pixel size that was used to discretize this function.

In order to test the effects of the sizes of the pixels on the reconstructed density, we have performed an additional computation with a double number of pixels along $x_{1}$ and $x_{4}$. That is, the additional computation used a grid of $64 \times 64 \times 256 \times 64$ pixels, and the resulting density is denoted by $\rho_{\text {calc }}^{\prime \text { MEM }}$. Modulation functions were derived from $\rho_{\text {calc }}^{\prime \text { MEM }}$ by the procedure described above. The result showed that the agreement between the model and the reconstructed modulation functions along $x_{1}$ ( $x$ of the first subsystem) and $x_{4}$ ( $x$ of the second subsystem) has improved considerably (Table 4). In fact, the difference between these two has become less than half the value it was before (Table 5), in accordance with the double resolution along these directions.

We have thus shown that the major part of the discrepancies between the reconstructed density and the model is due to the discrete nature of $\rho^{\mathrm{MEM}}$ and that the accuracy of the modulation functions is limited to a fraction less than about $10 \%$ of the size of the pixels. Nevertheless, this finding still leaves several possibilities for the dependence of the result on the pixel size. It can be due to the fact that $\rho^{\mathrm{MEM}}$ does not represent the values of the electron density on the grid points but that it represents some type of average density, where the average involves all values of $\rho$ within the space around the grid point. Alternatively, the problem can lie in the method of interpolation that was used to obtain the values of $\rho^{\mathrm{MEM}}$ between the grid points.

Even if a sufficiently fine grid had been selected, sources of error remain. They include

(i) An inaccuracy of the MaxEnt algorithm resulting in an electron density that is not the density with the maximum value of the entropy.

(ii) An inaccuracy that is intrinsic to the method. Only a finite number of reflections can be used. This causes seriestermination effects and the so-called aliasing effect, resulting in artifacts and noise in $\rho^{\mathrm{MEM}}$ (Jauch, 1994; Roversi et al., 1998; Palatinus \& van Smaalen, 2002).

(iii) Problems related to the estimated standard uncertainties. Although the calculated data are noise-free, the MEM requires that non-zero standard uncertainties be assigned to them. Therefore, the MEM will never converge to a perfect fit to the data.

At present, we do not have a quantitative estimate of the importance of these different effects. However, for the case of $(\mathrm{LaS})_{1.14} \mathrm{NbS}_{2}$ with the extensive data set that was available to us, the sources of error listed above apparently are less important than the effects of the limited resolution.

The reconstructed electron density $\rho_{\mathrm{obs}}^{\mathrm{MEM}}$ may show features that are not described by the model. The analysis shows that the modulation functions derived from $\rho_{\text {obs }}^{\mathrm{MEM}}$ follow the model quite well and in particular they match the modulation func- 
tions derived from $\rho_{\text {calc }}^{\mathrm{MEM}}$ very well (Fig. 7). The differences between $\rho_{\text {obs }}^{\mathrm{MEM}}$ and the model are larger than the differences between $\rho_{\text {calc }}^{\mathrm{MEM}}$ and the model but they still represent a small fraction of the pixel size only. In this respect, it should be noted that additional sources of error are present when the MEM is applied to the experimental data instead of the calculated data. They include the following points:

(iv) The data contain noise. Although it is known that the MEM operates as a noise filter (Skilling \& Bryan, 1984), the optimized density might still be different from the one obtained with noiseless data.

(v) The standard uncertainties of the data contain noise.

(vi) Both the standard uncertainties and the measured amplitudes may contain systematic deviations from their true values because of systematic errors, like an absorption correction or extinction correction that is not perfect.

Point (iv) particularly applies to the satellite reflections. About half of them are less thans and the MEM cannot fit their noisy values (Table 3 ). On the other hand, all of these reflections do have correct values in the calculated data. The discrepancies between the modulation functions derived from the calculated data and those derived from the observed data will thus be heavily affected by the many satellites for which significant intensity values are missing. Although these sources of error may also affect the values of the parameters in the model that has been refined, the two methods are affected in different ways by errors in the data and differences between the two are expected accordingly.

In view of (i)-(vi) and the pixel-size effect, we believe that the major part of the differences between the model and the reconstructed modulation functions is artifacts and noise. However, in estimating the reliability of the reconstructed modulation functions, one also needs to take into account the scattering powers of the individual atoms. For the weaker scatterers S1 and S2, we believe that the differences between the modulation functions derived from $\rho_{\text {obs }}^{\mathrm{MEM}}$ and $\rho_{\text {calc }}^{\mathrm{MEM}}$ are indeeed artifacts and noise. But for $\mathrm{Nb}$ it is found that modulation functions of the model are followed by the reconstructed functions much better than for the $\mathrm{S}$ atoms,

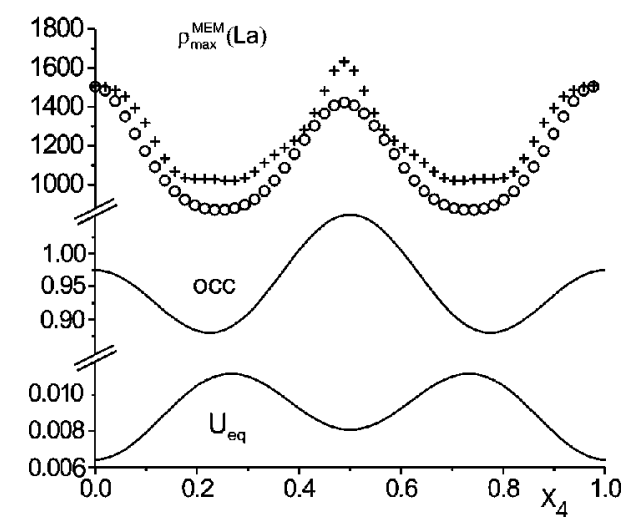

Figure 8

Modulation of the temperature factor $\left(U_{\text {eq }}\right)$ and the occupancy of $\mathrm{La}$ (occ) compared with the values of $\rho_{\text {calc }}^{\mathrm{MEM}}$ (circles) and $\rho_{\mathrm{obs}}^{\mathrm{MEM}}$ (crosses) at the positions of the La atoms $\left[\rho_{\max }^{\mathrm{MEM}}(\mathrm{La})\right]$. although the displacements of $\mathrm{Nb}$ are smaller than for $\mathrm{S} 1$ and $\mathrm{S} 2$. Noise is visible but it is an order of magnitude smaller than for S1 and S2. Lanthanum is the strongest scatterer, and it is thus tempting to interpret the deviations between model and reconstructed functions as a true structural effect. This is especially so for the displacements along $x_{3}$. Although we believe that the reconstructed modulation does indicate that there are deviations from the harmonic model, we refrain from a detailed analysis. We maintain our interpretation that the modulations in $(\mathrm{LaS})_{1.14} \mathrm{NbS}_{2}$ are described well by modulation functions based on the combinations of two harmonics (Fig. 7).

We have identified the positions of the atoms with the maxima in the electron density. In the case of anharmonic temperature movements, the electron density is asymmetric and its maximum does not need to coincide with the position of the atom. For $(\mathrm{LaS})_{1.14} \mathrm{NbS}_{2}$, both the refinements and the reconstructed electron density do not give any indication for anharmonic temperature factors and we believe that this source of error in deriving the displacement modulations is not important here.

Other effects that were important for the successful modelling of the structure were the modulations of the harmonic temperature factors and the occupancy of the La site (Jobst \& van Smaalen, 2002). Both modulations lead to a modulation of the value of the electron density along the trace of its maximum as a function of $t$. For both $\rho_{\text {calc }}^{\mathrm{MEM}}$ and $\rho_{\text {obs }}^{\mathrm{MEM}}$, the values of the electron density at the positions of La indeed exhibit a variation with $t$, which matches with the modulations of the temperature factors and the occupancy (Fig. 8). It can be concluded that the MEM is able to reconstruct these aspects of the modulations too.

\section{Conclusions}

The maximum-entropy method is critically evaluated for application to incommensurately modulated structures and incommensurate composite crystals. An efficient algorithm is described that allows the complete superspace group symmetry to be used ( $\S 2.2-2.4)$.

The method has been implemented into a computer program, BayMEM, that performs MaxEnt optimization of the electron density in superspace against phased structure factors. It uses the full space group or superspace group symmetry and it allows computations in superspace of arbitrary dimensions. Periodic crystals are treated as the special case with $d=0$.

Numerical applications to the scattering data of the periodic crystals silicon, $\mathrm{NaV}_{2} \mathrm{O}_{5}$ and oxalic acid have revealed the following features.

The Cambridge and Sakata-Sato algorithms lead to similar results for $\rho^{\mathrm{MEM}}$. The differences between the two algorithms are less than the apparent noise in the resulting densities (§3.2). The Cambridge algorithm converges faster and more reliably than the Sakata-Sato algorithm, when the $F_{2}$ constraint is used. 
Applications of the MEM to problems of intermediate and large sizes (e.g. $\left.\mathrm{NaV}_{2} \mathrm{O}_{5}\right)$ require the use of efficient algorithms for the Fourier transform and the handling of symmetry.

BayMEM has been used to study the modulation functions in the crystal structure of the inorganic misfit layer compound $(\mathrm{LaS})_{1.14} \mathrm{NbS}_{2}$. This compound belongs to the class of incommensurate composite crystals, and its structure is described in (3+1)-dimensional superspace. It has been shown that:

The MEM is able to reconstruct the modulation functions for the displacement modulations of the atoms. $\rho^{\mathrm{MEM}}$ even shows features that can be identified with the modulations of the temperature factors and the occupancy of the La site.

The accuracy is limited to a fraction of about $10 \%$ of the sizes of the pixels. This represents the major problem for the accurate determination of small modulation amplitudes, because the pixel size is limited by the amount of computational power that is available. For $d=1$, an increase of the resolution by a factor of two along each direction in superspace implies an increase of the computational time by about a factor of 16. At present, the resolution that can be attained is of the order of $0.1 \AA$, thus limiting the accuracy to about $0.01 \AA$.

The quality of the MaxEnt reconstruction is negatively affected when many satellite reflections are of the type less than. Improvements to the reconstruction can be expected when a method will be developed for designing a non-uniform prior in superspace.

Finally, it is noticed that the purpose of the application of the MEM to aperiodic crystals is to construct modulation functions in cases where they cannot be modelled by a few harmonics. This has been done successfully for the incommensurate composite crystal structure of the high-pressure phase III of bismuth, as will be reported elsewhere (McMahon et al., 2003).

Financial support by the Deutsche Forschungsgemeinschaft (DFG) is gratefully acknowledged.

\section{References}

Bagautdinov, B., Luedecke, J., Schneider, M. \& van Smaalen, S. (1998). Acta Cryst. B54, 626-634.

Becker, P. \& Coppens, P. (1974). Acta Cryst. A30, 129-147.

Boissieu, M. de, Papoular, R. J. \& Janot, C. (1991). Europhys. Lett. 16, 343-347.

Collins, D. M. (1982). Nature (London), 298, 49-51.

Dinnebier, R. E., Schneider, M., van Smaalen, S., Olbrich, F. \& Behrens, U. (1999). Acta Cryst. B55, 35-44.

Engel, P. (1986). Geometric Crystallography. Dordrecht: Reidel.

Gilmore, C. J. (1996). Acta Cryst. A52, 561-589.

Gull, S. F. \& Skilling, J. (1999a). MEMSYS5 v1.2 Program Package. Maximum Entropy Data Consultants Ltd, Suffolk, England.

Gull, S. F. \& Skilling, J. (1999b). Quantified Maximum Entropy, MEMSYS5 Users' Manual. Maximum Entropy Data Consultants Ltd, Suffolk, England.

Hahn, T. (1995). Editor. International Tables for Crystallography, Vol. A. Dordrecht: Kluwer Academic Publishers.
Haibach, T., Cervellino, A., Estermann, M. A. \& Steurer, W. (2000). Z. Kristallogr. 215, 569-583.

Jauch, W. (1994). Acta Cryst. A50, 650-652.

Jobst, A. \& van Smaalen, S. (2002). Acta Cryst. B58, 179-190.

Kumazawa, S., Kubota, Y., Takata, M., Sakata, M. \& Ishibashi, Y. (1993). J. Appl. Cryst. 26, 453-457.

Kumazawa, S., Takata, M. \& Sakata, M. (1995). Acta Cryst. A51, 651-658.

Linden, W. von der, Dose, V., Fisher, R. \& Preuss, R. (1998). Editors. Maximum Entropy and Bayesian Methods. Dordrecht: Kluwer Academic Publishers.

McMahon, M. I., Degtyareva, O., Nelmes, R. J., van Smaalen, S. \& Palatinus, L. (2003). In preparation.

Palatinus, L. \& van Smaalen, S. (2002). Acta Cryst. A58, 559-567.

Papoular, R. J., de Boissieu, M. \& Janot, C. (1991). In Methods of Structural Analysis of Modulated Structures Quasicrystals, edited by J. M. Perez-Mato, F. J. Zuniga \& G. Madariaga, pp. 333-343. Singapore: World Scientific.

Papoular, R. J., Collin, G., Colson, D. \& Viallet, V. (2002). In Proccedings of the 21st Workshop on Bayesian Inference and Maximum Entropy Methods in Science and Engineering, edited by B. Fry. Melville, NY: American Institute of Physics.

Perez-Etxebarria, A., Madariaga, G. \& Elcoro, L. (2001). Ferroelectrics, 250, 413-416.

Petricek, V. \& Dusek, M. (1998). JANA98 Computer Programs. Institute of Physics, Praha, Czech Republic.

Petricek, V. \& Dusek, M. (2000). The Crystallographic Computing System JANA2000. Institute of Physics, Praha, Czech Republic.

Press, W. H., Teukolsky, S. A., Vetterling, W. T. \& Flannery, B. P. (1996). Numerical Recipes in Fortran 77, 2nd ed. Cambridge University Press.

Roversi, P., Irwin, J. J. \& Bricogne, G. (1998). Acta Cryst. A54, 971-996.

Saka, T. \& Kato, N. (1986). Acta Cryst. A42, 469-478.

Sakata, M. \& Sato, M. (1990). Acta Cryst. A46, 263-270.

Sakata, M. \& Takata, M. (1996). High Press. Res. 14, 327-333.

Schneider, M. (2001). PhD thesis, University of Bayreuth, Germany.

Schneider, M. \& van Smaalen, S. (2000). Acta Cryst. A56, $248-251$.

Skilling, J. \& Bryan, R. K. (1984). Mon. Not. R. Astron. Soc. 211, 111-124.

Smaalen, S. van (1991). J. Phys. Condens. Matter, 3, 1247-1263.

Smaalen, S. van (1995). Crystallogr. Rev. 4, 79-202.

Smolinski, H., Gros, C., Weber, W., Peuchert, U., Roth, G., Weiden, M. \& Geibel, C. (1998). Phys. Rev. Lett. 80, 5164-5167.

Steurer, W. (1991). In Methods of Structural Analysis of Modulated Structures Quasicrystals, edited by J. M. Perez-Mato, F. J. Zuniga \& G. Madariaga, pp. 344-349. Singapore: World Scientific.

Su, Z. \& Coppens, P. (1997). Acta Cryst. A53, 749-762.

Takata, M. \& Sakata, M. (1996). Acta Cryst. A52, 287-290.

Vries, R. Y. de, Briels, W. J. \& Feil, D. (1994). Acta Cryst. A50, 383-391.

Vries, R. Y. de, Briels, W. J. \& Feil, D. (1996). Phys. Rev. Lett. 77, 1719-1722.

Wang, C.-R., Tsutomu, K., Tomiyama, T., Yoshida, T., Kobayashi, Y., Nishibori, E., Takata, M., Sakata, M. \& Shinohara, H. (2001). Angew. Chem. Int. Ed. Engl. 40/2, 397-399.

Weber, S. \& Yamamoto, A. (1997). Philos. Mag. A, 76, 85-106.

Wiegers, G. A. (1996). Prog. Solid State Chem. 24, 1-139.

Wolff, P. M. de, Janssen, T. \& Janner, A. (1981). Acta Cryst. A37, 625-636.

Yamamoto, A., Weber, S., Sato, A., Kato, K., Ohshima, K.-i., Tsai, A. P., Niikura, A., Hiraga, K., Inoue, A. \& Masumoto, T. (1996). Philos. Mag. Lett. 73, 247-254. 